\title{
Liquid carbon: structure near the freezing line
}

\author{
Luca M Ghiringhelli ${ }^{1}$, Jan H Los ${ }^{2}$, Evert Jan Meijer ${ }^{1}$, A Fasolino ${ }^{1,2}$ and \\ Daan Frenkel ${ }^{1,3}$ \\ ${ }^{1}$ van 't Hoff Institute for Molecular Sciences, Universiteit van Amsterdam, Nieuwe Achtergracht \\ 166, 1018 WV Amsterdam, The Netherlands \\ 2 Institute for Molecules and Materials, Radboud University Nijmegen, Toernooiveld 1, \\ 6525 ED Nijmegen, The Netherlands \\ ${ }^{3}$ FOM Institute for Atomic and Molecular Physics, Kruislaan 407, 1098 SJ, Amsterdam, \\ The Netherlands
}

Received 16 September 2005

Published 28 October 2005

Online at stacks.iop.org/JPhysCM/17/S3619

\begin{abstract}
We present a detailed analysis of the structure of liquid carbon near the freezing line. The results are obtained by molecular simulation using a recently developed state-of-the-art bond order potential. We find that along the melting line the liquid is predominantly threefold coordinated up to pressures far beyond the liquid-graphite-diamond triple point. We find no sign of a first-order liquidliquid phase transition when, at $10500 \mathrm{~K}$ and $\sim 300 \mathrm{GPa}$, the local structure of the liquid along the melting line changes dominant coordination from three- to fourfold.
\end{abstract}

(Some figures in this article are in colour only in the electronic version)

\section{Introduction}

Knowledge of the phase diagram of carbon under extreme conditions is of crucial importance for a better understanding of a wide variety of physical phenomena. For example, the phase diagram determines the carbon content of the interior of the Earth and other planets, and it governs the optimal conditions for the manufacturing of synthetic diamonds. In spite of intensive experimental and theoretical investigations [1-15], knowledge of the phase diagram of carbon for pressures $(P)$ and temperatures $(T)$ in the range up to $100 \mathrm{GPa}$ and $10000 \mathrm{~K}$ is still fragmented because experiments under these conditions are difficult if not outright impossible. Until recently, accurate quantitative theoretical and numerical predictions were hampered by the fact that the existing atomistic models for carbon had serious flaws that made them unsuited for quantitative predictions. Some of us developed a model potential for carbon, the long range carbon bond order potential (LCBOP) [16], that provides an accurate description of the solid phases. Recently, we have modified that model to properly describe the liquid phase also [17]. We have shown that the potential reproduces accurately $a b$ initio and experimental results of 
various properties of carbon materials [16-18]. These include the structure and energetics of small carbon clusters, the interplanar interactions in graphite, the graphite-diamond melting line, and the structure of liquid carbon. Hence, the LCBOP is the first empirical potential that is capable of providing an accurate description of graphite, diamond, and liquid carbon. At pressures and temperatures much higher than considered here, carbon may form structures with coordination numbers beyond four [19]. Proper modelling of that region of the phase diagram may require an adaptation of the LCBOP.

Employing the LCBOP we have determined, by means of free-energy calculations, the carbon phase diagram with unprecedented accuracy [18]. In this paper, we present a detailed analysis of the structure of the liquid near the freezing line, both in the stable and meta-stable region. In the range of pressures and temperatures up to $100 \mathrm{GPa}$ and $10000 \mathrm{~K}$, carbon exhibits a graphite and a diamond solid phase at lower temperatures, and a liquid phase at higher temperatures (figure 1). The graphite-diamond coexistence line is experimentally well accessible, and has been properly characterized up to $2400 \mathrm{~K}[1,8]$ by means of direct conversion experiments at equilibrium. In contrast, the experimental determination of the melting lines of graphite and diamond is rather uncertain due to the extreme conditions. For the graphite melting line a large number of rather scattered experimental data are available $[2,3,9,4,6,7]$. Reported values for the melting temperature at atmospheric pressure range from 3700 to $5000 \mathrm{~K}$, and appear to be strongly dependent on the heating rate of the sample $[6,7]$. All the experiments indicate that the melting temperature varies little with pressure, and most of them show a graphite melting $P-T$ line $[2,3,9]$ that has a maximum at $P \sim 6 \mathrm{GPa}$. However, the nature of the maximum is not well established. This is important as a point on the melting curve with a discontinuous change of the slope would be the starting point of a liquid-liquid phase transition (LLPT) line. The possible existence of an LLPT for carbon has been the subject of much speculation. First theoretically predicted $[10,11]$ on the basis of a pseudobinary solution theory [20], the LLPT for carbon was indeed found for the semi-empirical Brenner bond-order potential [21] by computer simulation [13]. Subsequently, this LLPT was demonstrated [15, 17] to be spurious due to shortcomings of the Brenner potential. For the diamond melting line few experimental data are available. Shock wave experiments [5] (solid circle in figure 1) indicated that at $P=140 \mathrm{GPa}$ the diamond sample was not yet melted at a temperature beyond the triple-point temperature. This implies that the carbon diamond melting line has a positive slope in the $P-T$ diagram. The diamond melting line for the Brenner bond-order potential (dashed line in figure 1), obtained by computer simulation [14], also shows a positive slope. However, comparison with the shock wave experiment at $140 \mathrm{GPa}$ indicates that the Brenner model underestimates the diamond melting temperature.

\section{Phase diagram}

The phase diagram was calculated by locating the points with equal chemical potential in the $P-T$ phase diagram. The details of the calculation are given elsewhere [18]. The calculated phase diagram is shown in figure 1 for the full range of pressures and temperatures considered. The three coexistence lines meet in a triple point at $16.4 \pm 0.7 \mathrm{GPa}$ and $4250 \pm 10 \mathrm{~K}$. The graphite-diamond coexistence line agrees very well with the experimental data [8]. The calculated graphite melting line increases monotonically in a small temperature range around $4000 \mathrm{~K}$. In contrast to data inferred from experiments it shows no maximum and is at a somewhat lower temperature. In agreement with the experiments the coexistence temperature is only slowly varying with pressure. Inspection reveals that this behaviour is due to (1) the limited variability of the melting enthalpy and (2) a similar bulk modulus for liquid and graphite such 


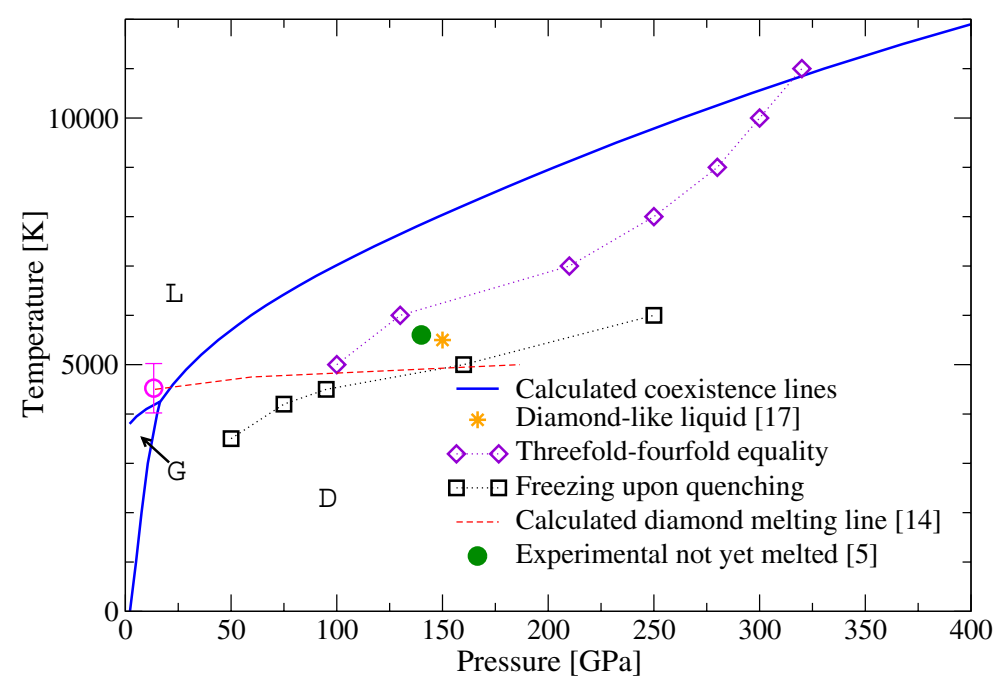

Figure 1. Phase diagram of carbon. The solid line indicates the calculated LCBOP phase boundaries, as reported in [18], between the liquid (L), graphite (G), and diamond (D). The circle with the error bar represents the graphite-diamond-liquid triple point, as extrapolated from experimental data [8]. The solid circle is the final point of the shock wave experiment of [5] at which diamond is not yet melted. Diamonds mark the liquid with equal amount of three and fourfold atoms. Squares represent state points in which the sample freezes. The asterisk in the region enclosed between these two series is a 'diamond-like liquid' reported on in [17]

that the difference in specific volume between the two phases $(\Delta v)$ is almost constant. The slope of the diamond melting line is consistent with the only experimental point available [5]. When compared to the diamond melting line of the Brenner model [14], the LCBOP diamond melting line has a steeper slope, yielding significantly higher temperatures for the diamond melting line. The most important difference with the phase diagram of the Brenner potential is that the LCBOP exhibits no liquid-liquid phase transition near the graphite melting line [13]. This is consistent with recent ab initio [15, 17] and tight-binding [22] molecular dynamics simulations. Note that the spurious LLPT of the Brenner potential involved a transition from a mainly twofold to a mainly fourfold coordinated liquid. Threefold coordinated atoms are virtually absent in the Brenner model due to an overestimation of the torsional interactions [15, 17]. As we will see below, the more accurate description of the torsional interactions implemented in LCBOP yields a mainly threefold liquid along the melting line. A fourfold coordinated liquid only appears in the high-pressure range.

\section{Liquid structure near coexistence}

Figure 2, left panel, shows the coordination fraction in the liquid along the melting lines, as a function of density, pressure and temperature, with the latter two appearing on a non-linear scale. On the left-hand side of the triple point, indicated by the dashed line, the liquid coexists with graphite, while on the right-hand side it coexists with diamond. The main observation is that for a large part of the melting line the liquid is dominantly threefold coordinated. In the graphite region, the three- and twofold coordination fractions remain rather constant, with almost no fourfold coordinated structures. Along the diamond melting line the threefold coordinated atoms are gradually replaced by fourfold coordinated atoms. Only at $300 \mathrm{GPa}$ does the liquid have an equal fraction of threefold and fourfold coordinated atoms. These results 

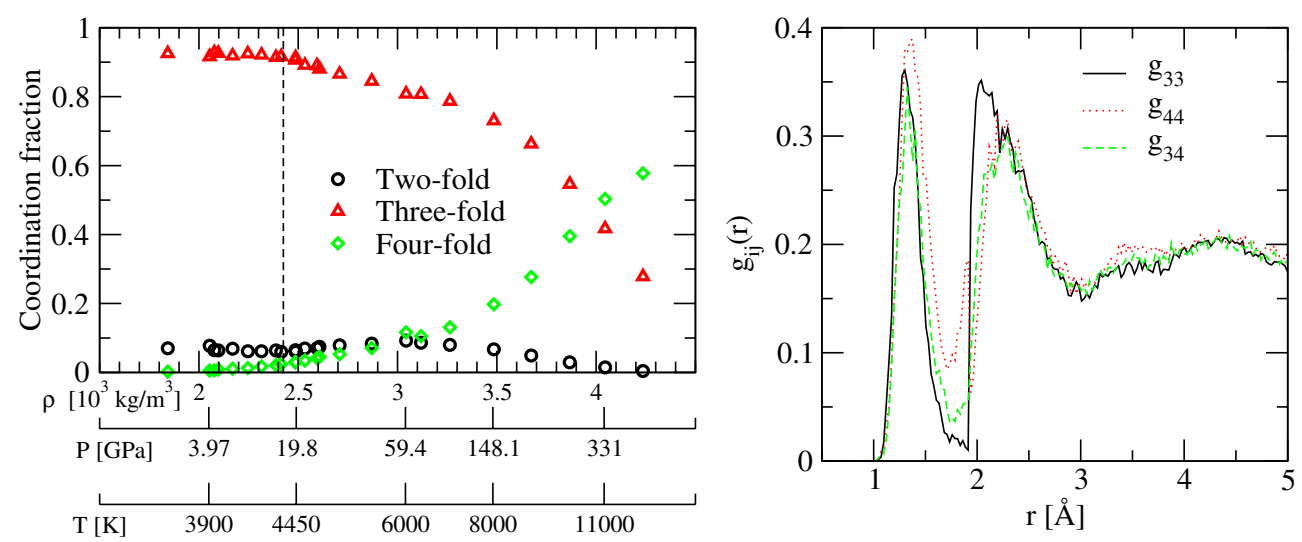

Figure 2. Left panel: coordination fraction of the liquid along the melting line(s). We chose the $\rho$ scale to be linear. The dashed line is the liquid-graphite-diamond triple point. Right panel: partial distribution functions $g_{i j}$ of the liquid on the melting line at $10500 \mathrm{~K}$ and $\sim 300 \mathrm{GPa}$.

contradict the generally assumed picture (see e.g. [11]) that diamond melts into a fourfold coordinated liquid.

The spatial correlation between carbon atoms with the same or different coordinations will be illustrated by the partial radial distribution functions (RDFs) $g_{i j}(r)$, defined as the probability of finding a $j$-fold site at a distance $r$ from a $i$-fold site. We considered the liquid at the point on the melting line where the liquid has an equal fraction of three- and fourfold coordinated atoms $(P=300 \mathrm{GPa}, T=10500 \mathrm{~K})$. Figure 2, right panel, shows the partial RDFs among threefold coordinated atoms $\left(g_{33}\right)$ and among the fourfold coordinated atoms $\left(g_{44}\right)$, and the cross RDFs among three- and fourfold coordinated atoms $\left(g_{34}\right)$. Except for the first minimum, the similarity between the three partial RDFs is striking. It shows that there is complete mixing, with the two sites almost indistinguishable, and almost all atoms coordinated by equal fractions of three- and fourfold coordinated atoms. A possible phase transition associated with the structural change of a three- to a fourfold coordinated liquid should yield some segregation of three- and fourfold coordinated sites. The partial RDFs show no sign of segregation. Hence, these results do not support an LLPT between three- and fourfold coordinated liquids in this region of the phase diagram.

The diamonds in figure 1 indicate the state points that exhibit an equal number of three- and fourfold coordinated atoms. They range from the high-temperature region where the liquid is thermodynamically stable down into the diamond region, where the liquid is metastable for the LCBOP. The squares indicate state points in which the LCBOP liquid freezes in the simulation. Our data suggest that a (meta)stable liquid with a dominantly fourfold coordination may only exist for pressures beyond $\sim 100 \mathrm{GPa}$. This could imply that the freezing of liquid into a diamond structure might be severely hindered for a large range of pressures beyond the liquid-graphite-diamond triple point. In [17] it is also pointed out that at $6000 \mathrm{~K}$ the equation of state shows a change of slope around the transition to the fourfold liquid. At even lower temperatures this feature becomes more pronounced. However, before these structural changes could possibly become a first order transition, the liquid freezes into a mainly fourfold coordinated amorphous structure, at a temperature of $\sim 4500 \mathrm{~K}$. This observation is consistent with quenching molecular dynamics simulations $[23,24]$ that yielded a tetrahedral amorphous carbon. In these simulations a mainly threefold liquid freezes into an almost completely fourfold amorphous structure. 


\section{Summary and outlook}

In summary, we have presented an overview of the liquid phase of carbon, as described by a new state-of-the-art bond-order potential (LCBOP). The calculated phase diagram for the LCBOP ruled out the existence of the hotly debated liquid-liquid phase transition in the region where the liquid is thermodynamically stable. Up to pressures of $300 \mathrm{GPa}$, the transformation into a dominantly fourfold liquid occurs in a region of the phase diagram where the liquid is metastable with respect to diamond. Beyond these pressures we have found no indication that the transformation of a mainly threefold to mainly fourfold coordinated liquid is associated with a first order phase transition.

In [17] we have presented the structure of a metastable liquid indicated by an asterisk in figure 1. We showed that this liquid has a 'diamond-like' structure, the first two peaks of the $\mathrm{RDF}$ of the liquid being very similar to that of diamond. Moreover, the angular distribution function of the first neighbours is strongly tetrahedral, and the angular correlation function for second neighbours shows a peak at $60^{\circ}$ also appearing in diamond. We consider this metastable 'diamond-like' liquid in this part of the phase diagram a good candidate for a quantitative study of crystallization into the diamond structure. At pressures around $100 \mathrm{GPa}$, this liquid is present at $\sim 20 \%$ undercooling, that is a value at which a comparison with simple liquids can be made. The diamond-like liquid is rather structured but very slowly diffusing: it is not easy to predict if these features combined would frustrate or enhance the nucleation rate. A comparison with the threefold liquid at lower, but not too different, pressures (so that the liquid is still metastable towards diamond) would also be of major interest.

\section{Acknowledgments}

This work is part of the research program of the Stichting voor Fundamenteel Onderzoek der Materie (FOM), which is financially supported by the Nederlandse Organisatie voor Wetenschappelijk Onderzoek (NWO). EJM acknowledges the Royal Netherlands Academy of Art and Sciences for financial support. We acknowledge support from the Stichting Nationale Computerfaciliteiten (NCF) and the Nederlandse Organisatie voor Wetenschappelijk Onderzoek (NWO) for the use of supercomputer facilities. LMG wishes to thank Sara Iacopini for valuable suggestions.

\section{References}

[1] Bundy F P, Bovenkerk H P, Strong H M and Wentorf R H Jr 1961 J. Chem. Phys. 35383

[2] Bundy F P 1963 J. Chem. Phys. 38618

[3] Fateeva N S and Vereshchagin L F 1971 Pis. Zh. Eksp. Teor. Fiz. 13157

[4] Whittaker A G 1978 Science 2004343

[5] Shaner J W, Brown J M, Swenson A C and McQueen R G 1984 J. Physique Coll. 45 C8-235

[6] Asinovskii E I, Kirillin A V and Kostanovskii A V 1997 High Temp. 35704

[7] Asinovskii E I, Kirillin A V, Kostanovskii A V and Fortov V E 1998 High Temp. 36716

[8] Bundy F P, Bassett W A, Weathers M S, Hemley R J, Mao H K and Goncharov A F 1996 Carbon 34141

[9] Togaya M 1997 Phys. Rev. Lett. 792474

[10] Korsunskaya I A, Kamenetskaya D S and Aptekar I L 1972 Fiz. Met. Metalloved. 34942 Korsunskaya I A, Kamenetskaya D S and Aptekar I L 1972 Phys. Met. Metallogr. 3439 (Engl. Transl.)

[11] van Thiel M and Ree F H 1993 Phys. Rev. B 483591

[12] Fried L E and Howard W M 2000 Phys. Rev. B 618734

[13] Glosli J N and Ree F H 1999 Phys. Rev. Lett. 824659

[14] Glosli J N and Ree F H 1999 J. Chem. Phys. 110441

[15] Wu C J, Glosli J N, Galli G and Ree F H 2002 Phys. Rev. Lett. 89135701 
[16] Los J H and Fasolino A 2003 Phys. Rev. B 68024107

[17] Ghiringhelli L M, Los J H, Meijer E J, Fasolino A and Frenkel D 2004 Phys. Rev. B 69 100101(R)

[18] Ghiringhelli L M, Los J H, Meijer E J, Fasolino A and Frenkel D 2005 Phys. Rev. Lett. 94145701

[19] Grumbach M P and Martin R M 1996 Phys. Rev. B 5415730

[20] Rapoport E 1967 J. Chem. Phys. 462891

[21] Brenner D W 1990 Phys. Rev. B 429458

Brenner D W 1992 Phys. Rev. B 461948 (erratum)

[22] Morris J C, Wang C Z and Ho K M 1995 Phys. Rev. B 524138

[23] Marks N A 2002 J. Phys.: Condens. Matter 142901

[24] Marks N A, Cooper N C, McKenzie D R, McCulloch D G, Bath P and Russo S P 2002 Phys. Rev. B 65075411 